\title{
Incidence and Prognostic Impact of DNMT3A Mutations in Korean Normal Karyotype Acute Myeloid Leukemia Patients
}

\author{
Sang Hyuk Park, ${ }^{1,2}$ Jae-Cheol Choi, ${ }^{3}$ Shine Young Kim, ${ }^{1,2}$ Jongyoun Yi, ${ }^{1,2}$ \\ Seung Hwan Oh, ${ }^{4}$ In-Suk Kim, ${ }^{5}$ Hyung-Hoi Kim, ${ }^{1,2}$ Chulhun Ludgerus Chang, ${ }^{5}$ \\ Eun Yup Lee, ${ }^{1,2}$ Moo-Kon Song, ${ }^{6}$ Ho-Jin Shin, ${ }^{6}$ and Joo Seop Chung ${ }^{6}$ \\ ${ }^{1}$ Department of Laboratory Medicine, Pusan National University School of Medicine, Pusan National University Hospital, \\ 179 Gudeok-ro, Seo-gu, Busan 602-739, Republic of Korea \\ ${ }^{2}$ Biomedical Research Institute, Pusan National University Hospital, 179 Gudeok-ro, Seo-gu, Busan 602-739, Republic of Korea \\ ${ }^{3}$ Department of Laboratory Medicine, Hanmaeum Hospital, 21 Woni-daero, 682 Beon-gil, Seongsan-gu, \\ Changwon-si, Gyeongsangnam-do 642-832, Republic of Korea \\ ${ }^{4}$ Department of Laboratory Medicine, Inje University College of Medicine, 75 Bokji-ro, Busanjin-gu, Busan 614-735, Republic of Korea \\ ${ }^{5}$ Department of Laboratory Medicine, Pusan National University School of Medicine, Pusan National University Yangsan Hospital, \\ 20 Geumo-ro, Mulgeum-eup, Yangsan-si, Gyeongsangnam-do 626-770, Republic of Korea \\ ${ }^{6}$ Division of Hematology-Oncology, Department of Internal Medicine, Pusan National University School of Medicine, \\ Pusan National University Hospital, 179 Gudeok-ro, Seo-gu, Busan 602-739, Republic of Korea
}

Correspondence should be addressed to Eun Yup Lee; eylee@pusan.ac.kr

Received 10 October 2014; Revised 22 December 2014; Accepted 22 December 2014

Academic Editor: Martin Bornhaeuser

Copyright (c) 2015 Sang Hyuk Park et al. This is an open access article distributed under the Creative Commons Attribution License, which permits unrestricted use, distribution, and reproduction in any medium, provided the original work is properly cited.

Background. DNA methyltransferase 3A (DNMT3A) mutation was recently introduced as a prognostic indicator in normal karyotype (NK) AML and we evaluated the incidence and prognostic impact of DNMT3A mutations in Korean NK AML patients. Methods. Total 67 NK AML patients diagnosed during the recent 10 years were enrolled. DNMT3A mutations were analyzed by direct sequencing and categorized into nonsynonymous variations (NSV), deleterious mutations (DM), and R882 mutation based on in silico analysis results. Clinical features and prognosis were compared with respect to DNMT3A mutation status. Results. Three novel (I158M, K219V, and E177V) and two known (R736H and R882H) NSVs were identified and the latter three were predicted as DMs. DNMT3A NSVs, DMs, and R882 mutation were identified in 14.9\%-17.9\%, $10.3 \%-10.4 \%$, and 7.5\% of patients, respectively. DNMT3A mutations were frequently detected in FLT3 ITD mutated patients $(P=0.054,0.071$, and 0.071 in NSV, DMs, and R882 mutation, resp.) but did not affect clinical features and prognosis significantly. Conclusions. Incidences of DNMT3A NSVs, DMs, and R882 mutation are $14.9 \%-17.9 \%, 10.3 \%-10.4 \%$, and $7.5 \%$, respectively, in Korean NK AML patients. DNMT3A mutations are associated with FLT3 ITD mutations but do not affect clinical outcome significantly in Korean NK AML patients.

\section{Introduction}

Acute myeloid leukemia (AML) is a clonal hematologic disorder exhibiting heterogeneous prognosis based on the results of cytogenetic and molecular aberration analysis $[1,2]$. Among patients with AML, approximately half of them are categorized into the intermediate prognosis group defined in the recent National Comprehensive Cancer Network (NCCN) guidelines [3]. In AML patients with intermediate prognosis group, most of them show normal karyotype (NK) and the prognosis of these patients generally depends on molecular aberrations and fms-related tyrosine kinase 3 gene internal tandem duplications (FLT3 ITD) which are detected in $30 \%$ of AML patients and possess poor prognosis regardless of mutation status in other genes [4-8], nucleophosmin (NPM1), and CCAAT/enhancer binding protein $\alpha$ (CEBPA) mutations which are detected in $50 \%$ and $13-15 \%$ of NK AML, respectively, and exhibit good prognosis [9-15] having been well established based on various previous studies. In addition, the mutations in isocitrate dehydrogenase (IDH)1 
and 2 genes, which are identified in $6.0 \%-15.9 \%$ of AML patients, were recently introduced as an unfavorable prognostic indicator in NPM1 mutated AML patients [16-19].

Besides these mutations described above, recent studies based on the whole-genome sequencing approach found a new prognostic marker of mutations in DNA methyltransferase $3 A$ (DNMT3A) gene involving DNA methylation, which is the most important epigenetic process in the regulation of gene expression and chromatin structural remodeling, in the patients with NK AML [20-23]. DNMT3A mutations have been reported in $18-22 \%$ of AML (29-34\% of CN-AML) [24-27] and previous studies showed that the mutations of DNMT3A in AML are frequently found in the patients with NPM1 mutations and have the mutation hotspot of arginine in 882th codon (R882) [20-27]. Some previous studies reported that the patients with DNMT3A mutations show inferior overall survival (OS) compared to those without mutations $[20,21,25]$ and another recent study reported a trend of more frequent relapse and inferior OS in the patients who achieved complete remission (CR) when they possess DNMT3A mutations [27]. However, in contrast to NPM1 and CEBPA mutations in which the prognostic value was confirmed and which were included as a provisional entity in the 2008 WHO classifications, the prognostic value of DNMT3A mutations as well as IDH1/2 mutations has not been confirmed up to present time. In addition, although two recent studies reported poor prognostic impact of DNMT3A mutations in Chinese and Taiwanese AML patients [28, 29], studies which analyzed the clinical relevance of DNMT3A mutations in Asian population are outnumbered compared to Western population and there have been no reports on the clinical impact of DNMT3A mutations in Korean AML patients.

In this study, we performed direct sequencing of all 23 exons in DNMT3A gene in 39 NK AML patients who were diagnosed at single tertiary hospital in Korea and analyzed the mutation characteristics. In addition, we performed in silico analysis to predict the deleterious effect of detected nonsynonymous variations on the protein function and structure and estimated the clinical relevance of DNMT3A mutations on the prediction of clinical course while including another 28 NK AML patients.

\section{Materials and Methods}

2.1. Patients Selection. A total of 60 NK AML patients who were diagnosed from January 2004 to July 2010 and treated in Pusan National University Hospital were initially recruited from the retrospective review of electronic medical record (EMR), and a total of 39 patients in whom the quality of extracted bone marrow (BM) DNA at diagnosis was sufficient to be analyzed by direct sequencing were finally enrolled in the first study cohort. In these 39 patients, direct sequencing of all 23 exons in DNMT3A gene was performed to analyze the incidence and distribution characteristics of all detected variations in DNMT3A gene. The median age and follow-up interval of first study cohort was 39.0 years (range: 11.0 years83.0 years) and 5.2 months (range: 0.0 months -72.0 months), respectively, and this cohort included 25 male (64.1\%) and 14 female $(35.9 \%)$ patients.

In addition, total $28 \mathrm{NK}$ AML patients who were diagnosed from August 2010 to March 2014 at the same hospital were additionally enrolled as second study cohort to evaluate the clinical and prognostic impact of mutation hotspot R882 in DNMT3A gene. In these patients, direct sequencing of $D N M T 3 A$ exon 23 was performed. The median age and follow-up interval of second study cohort were 28.0 years (range: 15.0 years-80.0 years) and 6.4 months (range: 0.1 months -42.6 months), respectively, and this cohort included 12 male (42.9\%) and 16 female (57.1\%) patients.

2.2. Patients Treatment. All these patients received induction chemotherapy with cytarabine $100 \mathrm{mg} / \mathrm{m}^{2} /$ day for seven days plus daunorubicin $45 \mathrm{mg} / \mathrm{m}^{2} /$ day for three days (the $\mathrm{AD}$ regimen) or cytarabine $100 \mathrm{mg} / \mathrm{m}^{2} /$ day for seven days plus idarubicin $12 \mathrm{mg} / \mathrm{m}^{2} /$ day for three days (the AI regimen). CR was defined by less than $5 \%$ of residual leukemic blasts in the BM aspirates and more than $20 \%$ of BM cellularity, normal maturation of all cellular components (erythrocytic, granulocytic, and megakaryocytic series) in the BM aspirates, normal values for absolute neutrophil counts $(>1,000 / \mu \mathrm{L})$ and platelet counts $(>100,000 / \mu \mathrm{L})$ after the first or second course of induction chemotherapy. Relapse was defined as the presence of $\geq 5 \%$ leukemic blasts in BM aspirates for patients who had previously achieved CR. Stem cell transplantation (SCT) after first CR achievement were considered as a final treatment modality depending on the patient's age, availability of a suitable donor, and FLT3 ITD mutation status.

As a prognosis indicator, CR, relapse, and death rates were used and both OS and event-free survival (EFS) were additionally calculated. OS was defined as the interval from diagnosis to death or last follow-up. EFS was defined as the interval from CR to relapse or death for patients who were relapsed or expired during follow-up or the interval from CR to last follow-up for surviving patients who were not relapsed during follow-up interval. If the patient underwent SCT, the CR date was replaced by the SCT date in the calculation of EFS. This study was approved by the institutional review board of author's institution.

2.3. Mutation Analysis of DNMT3A Gene. DNA samples were extracted from the unstained remnant smear slides of BM aspirates or chromosomal cell pellets obtained at diagnosis using DNA Blood Mini extraction kit (Qiagen, Hilden, Germany), as recommended by manufacturer. All 23 exons of DNMT3A gene were amplified with primers listed in Table 1. All forward and reverse primers had M13F binding tag (tgtaaaacgacggccagt) and M13R binding tag (caggaaacagctatgacc) on the $5^{\prime}$ ends of each primer. Polymerase chain reactions (PCR) were conducted with hot start procedure according to the manufacturer's instructions (i-StarTaq, INTRON Biotechnology, Sungnam, Korea) described below: an initial denaturation at $95^{\circ} \mathrm{C}$ for 5 minutes and by 30 cycles of following denaturation at $95^{\circ} \mathrm{C}$ for 30 seconds, annealing at $60^{\circ} \mathrm{C}$ for 30 seconds, and extension at $72^{\circ} \mathrm{C}$ for 30 seconds. 
TABLE 1: The forward and reverse primer sequences used in the amplification of all 23 exons in DNMT3A gene.

\begin{tabular}{|c|c|}
\hline Primer & Primer sequence \\
\hline$>$ DNMT02F & tgtaaaacgacggccagtTCCAAAGACCACGATAATTCCTTC \\
\hline$>$ DNMT03F & tgtaaaacgacggccagtCTGGAATGCTACACTGCTGGG \\
\hline$>$ DNMT04F & tgtaaaacgacggccagtACTTGGAGAAGCGGAGTGAGC \\
\hline$>$ DNMT05F & tgtaaaacgacggccagtGGATGTGTAAAGAAGGAGGAGGG \\
\hline$>$ DNMT06F & tgtaaaacgacggccagtACATTGTGTTTGAGGCGAGTGC \\
\hline$>$ DNMT07F & tgtaaaacgacggccagtCTAATTCCTGGAGAGGTCAAGGTG \\
\hline$>$ DNMT08F & tgtaaaacgacggccagtTCTTGCCTCATTCAGATGGAGC \\
\hline$>$ DNMT09F & tgtaaaacgacggccagtGTGCTTGCAAGTGTAAGCCTCG \\
\hline$>$ DNMT10F & tgtaaaacgacggccagtCTTGAGCCTGACCCATCTGC \\
\hline$>$ DNMT11F & tgtaaaacgacggccagtTTCCTGTCAGCCTGTAACTGACC \\
\hline$>$ DNMT12F & tgtaaaacgacggccagtTTATTGATGAGCGCACAAGAGG \\
\hline$>$ DNMT13F & tgtaaaacgacggccagtAGGGAGAGGCCCTTCGGTGG \\
\hline$>$ DNMT14F & tgtaaaacgacggccagtGGTCATGTCTTCAGGGCTTAGG \\
\hline$>$ DNMT15F & tgtaaaacgacggccagtTTTCCATTCCAGGTAGCACACC \\
\hline$>$ DNMT16F & tgtaaaacgacggccagtAGGGTGTGTGGGTCTAGGAGC \\
\hline$>$ DNMT17F & tgtaaaacgacggccagtGACTTGGGCCTACAGCTGACC \\
\hline$>$ DNMT18F & tgtaaaacgacggccagtATAGGACAGTGGTGTGGCTCG \\
\hline$>$ DNMT19F & tgtaaaacgacggccagtGACAGCTATTCCCGATGACCC \\
\hline$>$ DNMT20F & tgtaaaacgacggccagtGCCGGCGCTGTTTCATGC \\
\hline$>$ DNMT21F & tgtaaaacgacggccagtCCTTCCCGCTGTTATCCAGG \\
\hline$>$ DNMT22F & tgtaaaacgacggccagtTGGCATATTTGGTAGACGCATGAC \\
\hline$>$ DNMT23F & tgtaaaacgacggccagtTCCCAGTCCACTATACTGACGTCTC \\
\hline$>$ DNMT91F & tgtaaaacgacggccagtACAAAGAAAATGTTCCCTCССТCC \\
\hline$>$ DNMT92F & tgtaaaacgacggccagtAAATCTGGTATGGTGGAAATGGG \\
\hline$>$ DNMT02R & caggaaacagctatgaccTCССТСТCCCAGGCCAGA \\
\hline$>$ DNMT03R & caggaaacagctatgaccATACATCACTGCCATCGACAGG \\
\hline$>$ DNMT04R & caggaaacagctatgaccAAGCAGACCTTTAGCCACGACC \\
\hline$>$ DNMT05R & caggaaacagctatgaccGAACAGCTAAACGGCCAGAGG \\
\hline$>$ DNMT06R & caggaaacagctatgaccACTGAGGCCCATCACTTCTGG \\
\hline$>$ DNMT07R & caggaaacagctatgaccAGATGGAGAGAGGAGAGCAGGAC \\
\hline$>$ DNMT08R & caggaaacagctatgaccCCTGGGATCAAGAACCTTCCC \\
\hline >DNMT09R & caggaaacagctatgaccCCTGCACTCCAACTTCCAGG \\
\hline$>$ DNMT10R & caggaaacagctatgaccGCAGGTCATTCAAGTCCTGACC \\
\hline$>$ DNMT11R & caggaaacagctatgaccATGCAGGCCTCCTGGTGC \\
\hline$>$ DNMT12R & caggaaacagctatgaccTCCCATGTCATTCAAACCTTCC \\
\hline$>$ DNMT13R & caggaaacagctatgaccACACAGTCAGCCAGAAGGCCG \\
\hline$>$ DNMT14R & caggaaacagctatgaccTGCTACCTGGAATGGAAAGACC \\
\hline$>$ DNMT15R & caggaaacagctatgaccAGGCTCCTAGACCCACACACC \\
\hline$>$ DNMT16R & caggaaacagctatgaccGCTGTGAAGCTAACCATCATTTCG \\
\hline$>$ DNMT17R & caggaaacagctatgaccAAATGAAAGGAGGCAAGGGC \\
\hline$>$ DNMT18R & caggaaacagctatgaccTTCTTCCTGTCTGCCTCTGTCC \\
\hline$>$ DNMT19R & caggaaacagctatgaccTGCAGATGAGACAGGATGAAGC \\
\hline$>$ DNMT20R & caggaaacagctatgaccCCACTATGGGTCATCCCACCTGC \\
\hline$>$ DNMT21R & caggaaacagctatgaccCATCCTGCССТTCСТTCTCC \\
\hline$>$ DNMT22R & caggaaacagctatgaccTGGGAAATGCTTGATAAAACCCAC \\
\hline$>$ DNMT23R & caggaaacagctatgaccTCTCTCCATCCTCATGTTCTTGG \\
\hline >DNMT91R & caggaaacagctatgaccCCAGCACTAAGTCAGCATCTCCAG \\
\hline$>$ DNMT92R & caggaaacagctatgaccTGGAGTTCTTATGGATCACACCC \\
\hline
\end{tabular}

DNMT3A: DNA methyltransferase 3A; F: forward; R: reverse. 
A final extension was done at $72^{\circ} \mathrm{C}$ at 5 minutes on Verity96 well thermal cycler PCR system (Applied Biosystems Inc., Foster City, CA, USA).

PCR products were purified with AccuPrep kit (Bioneer, Daejeon, Korea) and were used in the sequencing reaction using BigDye Terminator v.3.1 Cycle Sequencing Ready Reaction kit (Applied Biosystems Inc., USA). Direct sequencing of all 23 exons was performed with ABI 3130 Genetic Analyzer (Applied Biosystems Inc., USA). The SeqScape software version 2.6 (Applied Biosystems Inc., USA) was used to align the obtained and reference sequences (NM_175629.2) and all detected nonsynonymous and synonymous variations were described according to the guidelines of the Human Gene Variation Society (HGVS; http://www.hgvs.org/).

\subsection{In Silico Analysis to Predict the Deleterious Effect of All} Detected Nonsynonymous Variations on the Protein Function and Structure. To predict the deleterious effect of novel and known sequence variations, all detected nonsynonymous variations were submitted to three web-based programs: Sorting Intolerant From Tolerent (SIFT, http://sift.jcvi.org/), Polyphen-2 (version 2.2.2, http://genetics.bwh.harvard.edu/ $\mathrm{pph} /$ ), and MutationTaster (http://www.mutationtaster .org/). The web-based database dbSNP (http://www.ncbi.nlm .nih.gov/projects/SNP/) was applied in the confirmation of known SNP and the novelty of detected nonsynonymous variations was validated by web-based database Catalogue of Somatic Mutations in Cancer (COSMIC, http://cancer .sanger.ac.uk/cancergenome/projects/cosmic/). Cn3D macromolecular structure viewer was used to annotate the mutation sites in 3D structure [30].

2.5. Comparison of Clinical Characteristics and Prognosis in Patients with respect to Mutation Status of DNMT3A Gene. To assess the clinical relevance of DNMT3A mutation analysis, the comparison of clinical characteristics and prognosis in NK AML patients with respect to mutation status of DNMT3A genes was performed. All patients were categorized into two subgroups with respect to nonsynonymous variation status of DNMT3A gene and both clinical features and prognosis were compared between two subgroups to evaluate the clinical relevance of nonsynonymous variations of DNMT3A gene. Subsequently, nonsynonymous variations which were predicted as deleterious mutation in at least two of three in silico analyses were recruited and identical comparisons were performed again between patients with deleterious mutations and those without deleterious mutations to further evaluate the clinical relevance of deleterious mutations in DNMT3A gene.

Finally, to evaluate the clinical relevance of DNMT3A R882 mutation hotspot, all patients were categorized into two subgroups with respect to DNMT3A R882 mutation status, and identical comparisons were performed again. Clinical data of patients were obtained from retrospective review of EMR and included age and gender, complete blood cell (CBC) counts, blasts (\%) in BM aspirates, FLT3 ITD and D835 mutation status (at diagnosis), and date of $\mathrm{CR}$, relapse, and death (during follow-up).
2.6. Multivariate Analysis of Overall Survival. For the identification of prognostic impact in DNMT3A mutations in association with other possible prognostic indicators such as FLT3 ITD mutation status, age, BM blast (\%), and SCT performance status, the multivariate analysis was performed when the clinical variables mentioned above were included as covariables.

2.7. Statistical Analysis. Pearson chi-square yest or Fisher's exact test (for small numbers less than 5) were applied to compare categorical variables (gender, CR rates, relapse rates, and death rates) between each two patient subgroups. MannWhitney $U$ test was used to compare continuous variables (age, CBC counts at diagnosis and interval from diagnosis to CR) between each two patient subgroups. Estimates of OS and EFS were generated using Kaplan-Meier methods and were compared using a log-rank test. Multivariate analyses of OS were performed with a Cox's proportional hazards model. For all analyses, tests were two-tailed and $P$ values $\leq$ 0.05 and $0.05-0.10$ were considered statistically significant and marginally significant, respectively. All calculations were performed using SPSS 13.0.1 for Windows (SPSS Inc, Chicago, IL, USA).

\section{Results}

3.1. Summary of Detected Variations in DNMT3A Gene. Among 39 patients included in the first study cohort, two known synonymous variations (c.27C $>\mathrm{T}$ (rs41284843, minor allele frequency $=0.0851$ ) and c.1266G $>$ A (rs2276598, minor allele frequency $=0.2337)$ ) and one novel synonymous variation (c.2043C>G) were detected in 5, 19 (including 2 homozygotes), and 6 patients (12.8\%, 48.7\%, and $15.4 \%)$, respectively.

Total five different nonsynonymous variations, which are all missense mutations, were detected in seven $(17.9 \%)$ patients and included two known mutations (c.2207G>A (p.Arg736His, COSM133737, detected in one patient) and c.2645G > A (p.Arg882His, COSM52946, detected in two patients)) and three novel mutations (c.474C $>\mathrm{G}$ (p.Ile158Met, detected in two patients), c.530A $>\mathrm{T}$ (p.Glu177Val, detected in one patient), and c.656A $>\mathrm{G}$ (p.Lys219Arg, detected in two patients)). All 7 patients with DNMT3A mutations harbored single mutation, except for one patient who showed both K219R and $\mathrm{R} 736 \mathrm{H}$ mutations simultaneously. All 2 known mutations $(\mathrm{R} 736 \mathrm{H}$ and $\mathrm{R} 882 \mathrm{H})$ induced protein change in the catalytic domain of DNMT3A protein which has critical role in methyltransferase activity, and all three novel mutations (I158M, E177V, and K219R) were involved in protein change at the regulatory domain of DNMT3A protein. These results are summarized in Figure 1.

Subsequently performed direct sequencing of DNMT3A exon 23 in 28 NK AML patients who were included in second study cohort detected additional three R882H mutations. When summarizing these results, the incidence of DNMT3A nonsynonymous variations was calculated to be $7 / 39$ (17.9\%) in the first study cohort and finally estimated to be $10 / 67$ $(14.9 \%)$ when adding data from 28 patients in the second 


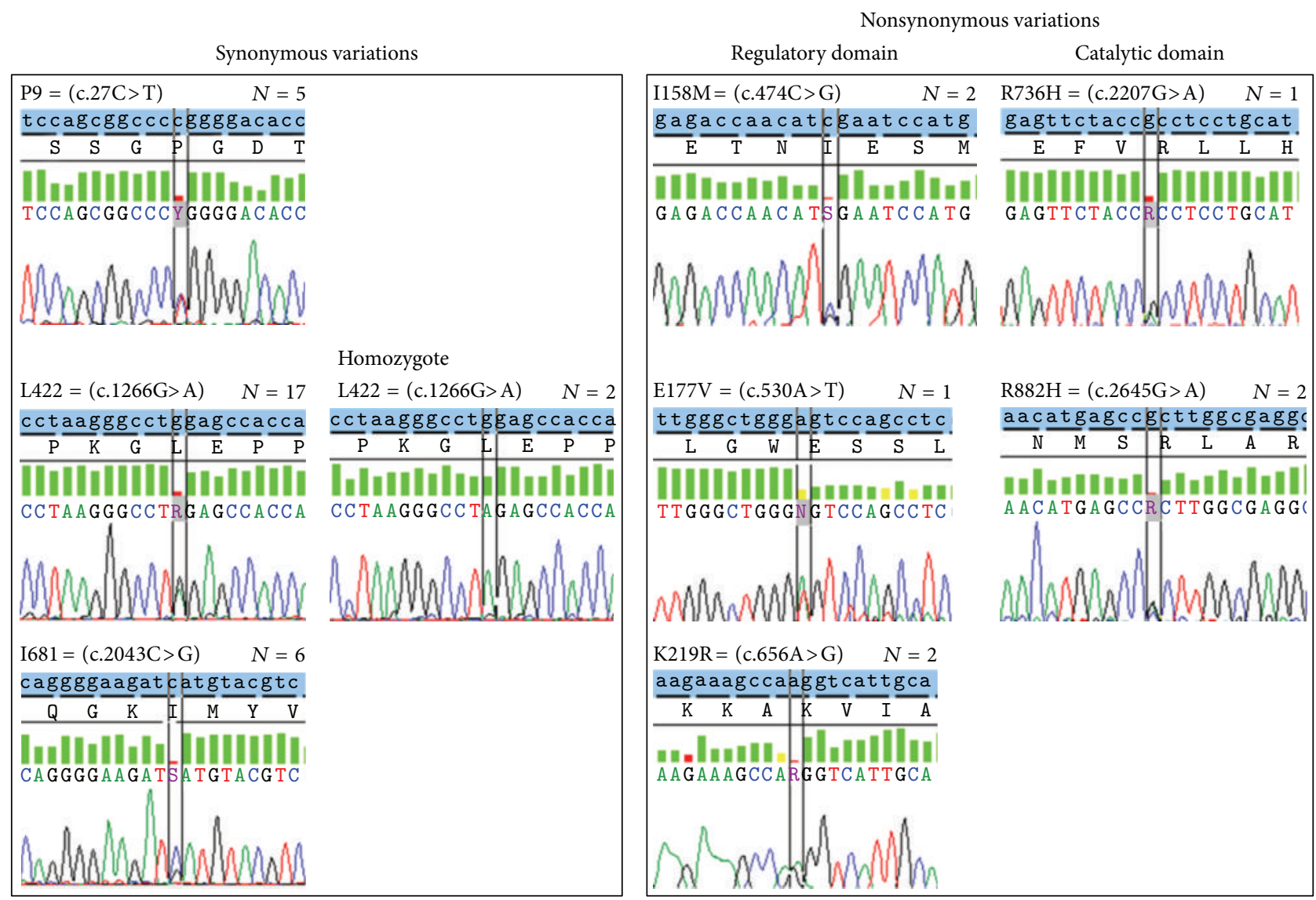

(a)

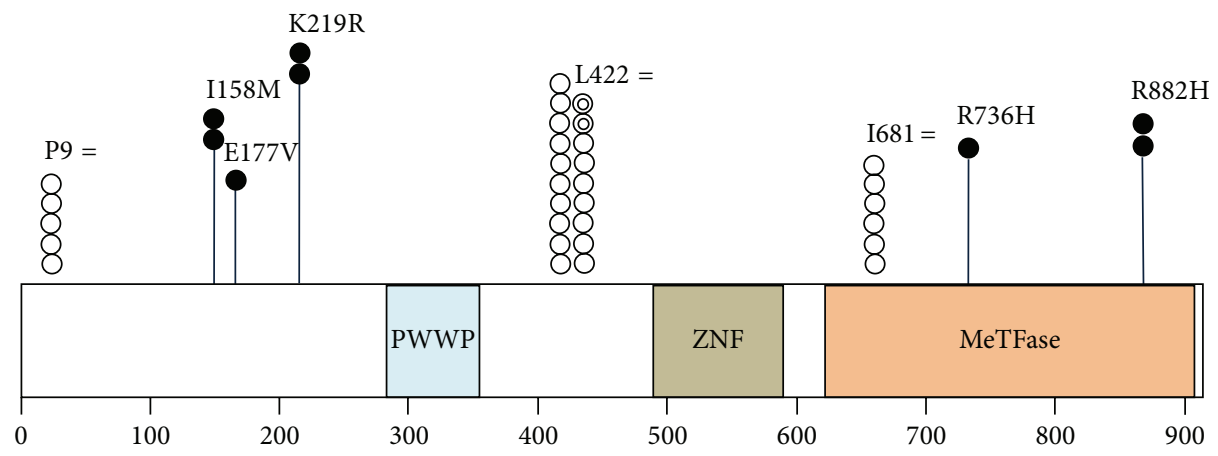

(b)

FIGURE 1: Electropherograms of all DNMT3A variations including synonymous and nonsynonymous variations observed in this study (a) and schematic summary representing locations and frequency of all detected DNMT3A variations (b). Note: (a) shows electropherogram of all detected DNMT3A variations in 39 patients included in the first study cohort. (b) shows schematic summary of all detected DNMT3A variation including location and frequency. The number of circles indicates the frequency of detected variations. Closed black and white circle indicates heterozygous nonsynonymous and synonymous variation case, respectively, and closed double-layered white circle indicates homozygous synonymous variation case. Abbreviations: P, proline; I, isoleucine; M, methionine; R, arginine; H, histidine; L, leucine; E, glutamate; V, valine; K, lysine; PWWP, Pro-Trp-Trp-Pro motif; ZNF, zinc finger; MeTFase, methyltransferase.

study cohort, and the frequency of DNMT3A R882 mutations was exactly calculated to be $5 / 67$ (7.5\%).

3.2. In Silico Analysis Results to Predict the Deleterious Effect of Detected Nonsynonymous Variations on the Protein Function and Structure. Among five DNMT3A nonsynonymous variations detected in this study, $\mathrm{R} 882 \mathrm{H}$ was predicted as a deleterious mutation by all three web-based prediction programs, as reported in the previous study [21] and another known variation $(\mathrm{R} 736 \mathrm{H})$ was also predicted as a deleterious mutation by two programs. In addition, one novel variation (E177V) was also predicted as a deleterious mutation by all three programs. In contrast, two novel variations (I158M and $\mathrm{K} 219 \mathrm{R}$ ) were predicted as a benign or tolerated variation in 
TABLE 2: In silico analysis results of three web-based programs to predict the deleterious effect of detected nonsynonymous variations on the protein function and structure.

\begin{tabular}{|c|c|c|c|c|c|c|c|}
\hline \multirow{2}{*}{ Mutations } & \multirow{2}{*}{$\begin{array}{l}\text { Sequence variation } \\
\text { database }\end{array}$} & \multicolumn{2}{|c|}{ SIFT } & \multicolumn{2}{|c|}{ Polyphen-2 } & \multicolumn{2}{|c|}{ MutationTaster } \\
\hline & & Score & Prediction & Score & Prediction & Score & Prediction \\
\hline $\mathrm{I} 158 \mathrm{M}$ & Novel & 0.21 & Tolerated & 0.191 & Benign & 0.27 & Polymorphism \\
\hline E177V & Novel & 0.02 & Damaging & 0.981 & Probably damaging & 3.30 & Disease causing \\
\hline K219R & Novel & 0.71 & Tolerated & 0.002 & Benign & 0.71 & Polymorphism \\
\hline $\mathrm{R} 736 \mathrm{H}$ & $\begin{array}{l}\text { rs139293773 } \\
\text { COSM133737 }\end{array}$ & 0.39 & Tolerated & 0.997 & Probably damaging & 0.79 & Disease causing \\
\hline $\mathrm{R} 882 \mathrm{H}$ & $\begin{array}{l}\text { rs147001633 } \\
\text { COSM52946 }\end{array}$ & 0.03 & Damaging & 0.651 & Possibly damaging & 0.79 & Disease causing \\
\hline
\end{tabular}

I: isoleucine; M: methionine; E: glutamate; V: valine; K: lysine; R: arginine; H: histidine.

all three programs. These results are represented in Table 2. With these results, the frequency of deleterious mutation of DNMT3A gene was calculated to be $4 / 39$ (10.3\%) in the first study cohort and finally estimated to be $7 / 67$ (10.4\%) when adding data from 28 patients in the second study cohort.

In the $3 \mathrm{D}$ structure model of methyltransferase domains, R882 residue was located in the region interfacing with two DNMT3A molecules, which is consistent with the results of previous literature [21], and R736 residue was located on the opposite side and toward DNMT3L molecule. These results are illustrated in the Supplemental Figure 1 available online at http://dx.doi.org/10.1155/2015/723682.

3.3. Comparison of Clinical Characteristics and Prognosis in Patients with respect to Mutation Status of DNMT3A Gene. In the comparison of patients with DNMT3A nonsynonymous variations and those without nonsynonymous variations, both clinical features and prognosis (OS and EFS) were not significantly different between two patient subgroups, except for the trend of high FLT3 ITD mutation frequency in patients with DNMT3A nonsynonymous variations than those who do not harbor (66.7\% versus $24.4 \%, P=0.054)$. Identical results were found in the comparison with respect to DNMT3A deleterious mutation status, since the patients with DNMT3A deleterious mutations also showed the trend of more frequent FLT3 ITD mutation than those without DNMT3A deleterious mutations $(75.0 \%$ versus $25.5 \%, P=$ $0.071)$. Additionally performed comparison between patients with DNMT3A R882 mutation and those without R882 mutation also showed identical results again. These results are summarized in Table 3 and Figure 2.

3.4. Multivariate Analysis of Overall Survival. The multivariate analysis of OS in total $67 \mathrm{NK}$ AML patients demonstrated that the presence of FLT3 ITD mutation is a significantly poor prognostic indicator of OS (hazard ratio 2.798, $P=$ 0.041) and the performance of SCT during follow-up is a significantly good prognostic indicator of OS (hazard ratio $0.118, P=0.001)$ as expected. However, the presence of DNMT3A nonsynonymous variations (hazard ratio 1.229, $P=0.724)$, deleterious mutations, and R882 mutations (hazard ratio $2.285, P=0.313$ in both) did not affect OS significantly as well as increasing age and BM blasts (\%), although the trend was toward to adverse prognosis. These results are described in Table 4.

\section{Discussion}

Previous studies that analyzed DNMT3A mutations showed that the incidence of DNMT3A mutation is $18-22 \%$ of AML and $29-34 \%$ of NK AML [24-27], including the largest study which analyzed 489 AML patients and reported the frequency of DNMT3A mutation as $17.8 \%$ of AML patients and $27.2 \%$ of NK AML patients [24]. In Korean population, although the data of clinical outcome was not provided, a recent study reported the incidence of DNMT3A R882 mutation as $9.4 \%$ in adulthood AML [31]. Our present study showed that the incidence of DNMT3A nonsynonymous variations is $17.9 \%$ in first study cohort and $14.9 \%$ in extended cohort, which is similar to the results from previous studies [24-27]. In addition, our present study analyzed the incidence of DNMT3A R882 mutation as 7.5\%, which is also similar to the previous data in Korean population [31]. All these results demonstrate that DNMT3A mutation can be also considered as a frequently occurring nonrandom mutation in Korean NK AML patients.

Our present study applied direct sequencing of all 23 exons in DNMT3A gene to identify all possible variations affecting DNMT3A protein function and structure and could detect total 8 different sequence variations, including 3 synonymous and 5 nonsynonymous variations. Among three synonymous variations, two known variations (c. $27 \mathrm{C}>\mathrm{T}$ and c. $1266 \mathrm{G}>\mathrm{A}$ ) of which minor allele frequencies are $8.51 \%$ and $23.37 \%$ were found with the frequency of $12.8 \%$ and $48.7 \%$, respectively, and therefore these two variations can be considered as constitutional single nucleotide polymorphisms (SNP). In addition, our present study could detect one novel synonymous variation (c.2043C $>\mathrm{G}$ ), with the incidence of $15.4 \%$ and this novel SNP can be listed in the current SNP databases.

For the nonsynonymous variations, we could find 3 different novel variations (I158M, E177V, and K219V) in the regulatory domains of DNMT3A protein that have a role in interaction with other molecules and protein localization to the nuclei, as well as 2 known variations ( $\mathrm{R} 736 \mathrm{H}$ and $\mathrm{R} 882$ ) located in the catalytic methyltransferase domain of 


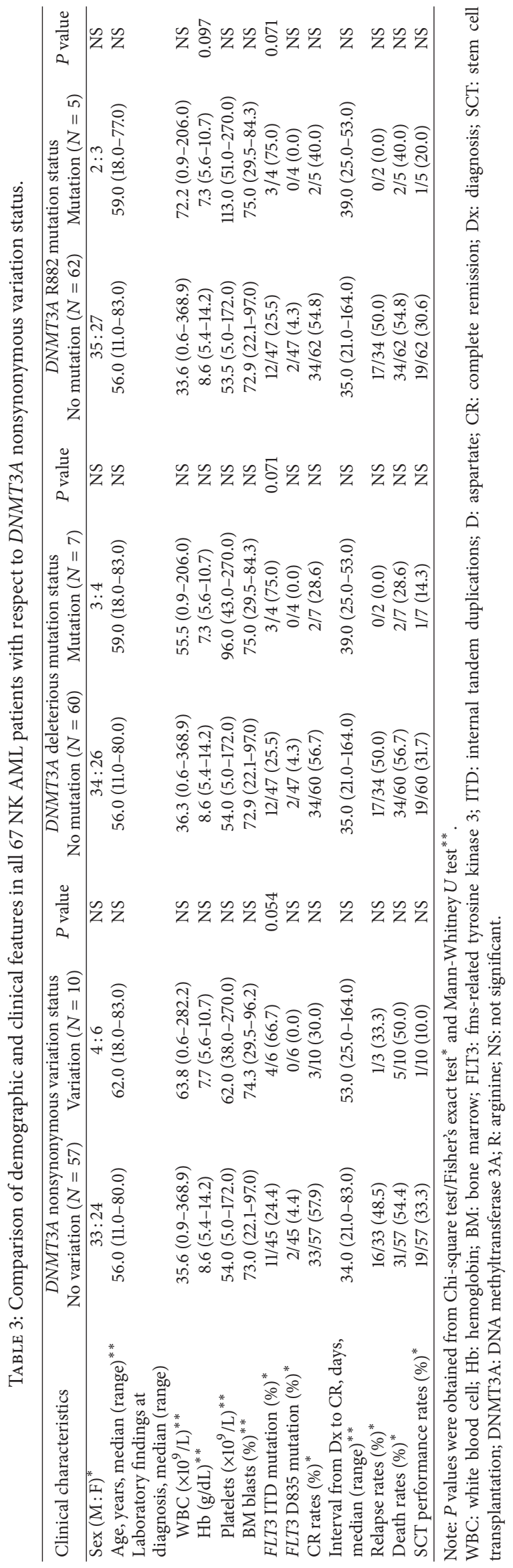




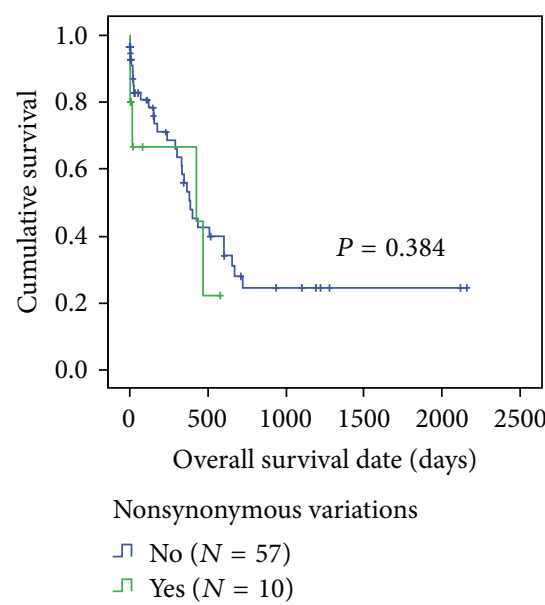

(a)

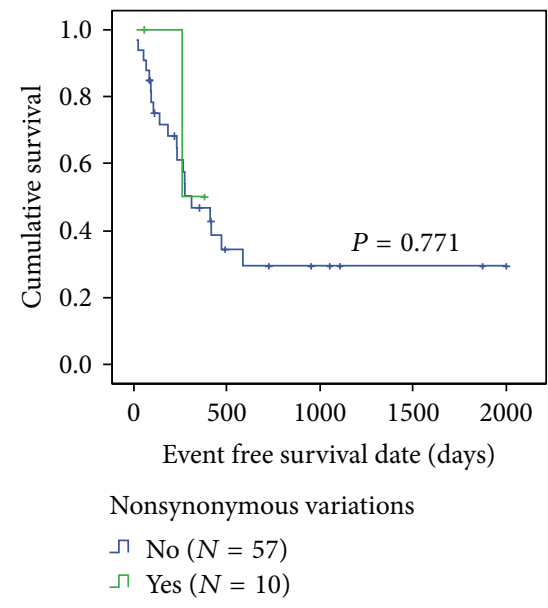

(d)

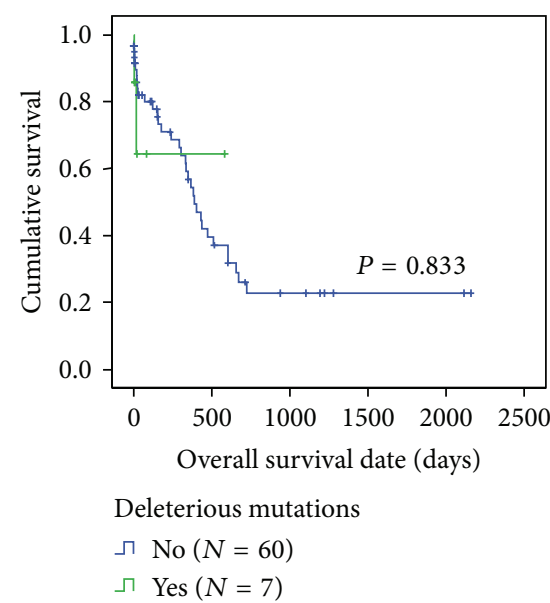

(b)

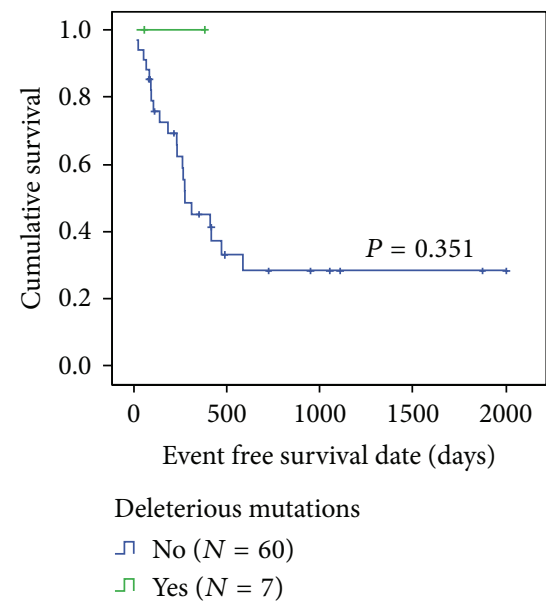

(e)

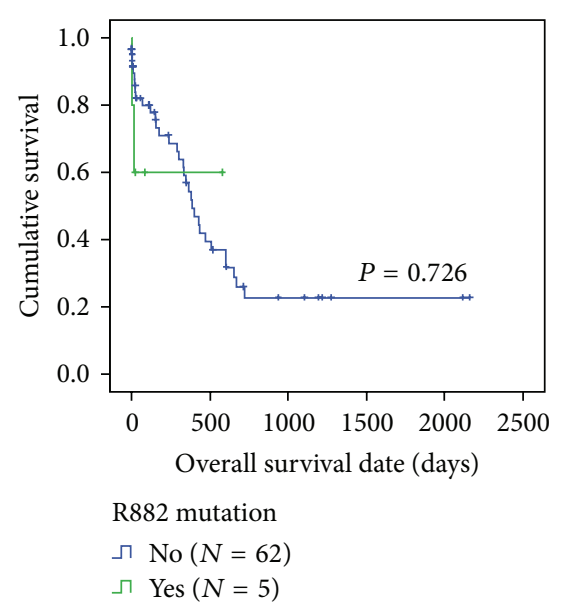

(c)

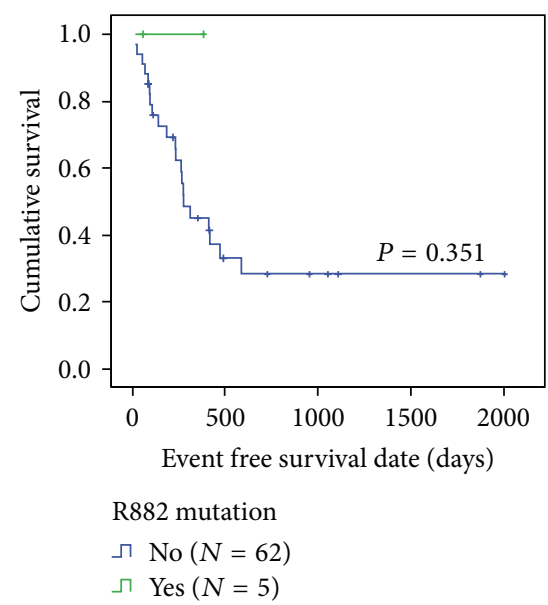

(f)

FIGURE 2: Comparisons of overall survival and event-free survival in total 67 NK AML patients with respect to DNMT3A nonsynonymous variations ((a) and (d)), deleterious mutations ((b) and (e)), and R882 mutation ((c) and (f)), respectively. Abbreviation: R, arginine.

DNMT3A protein. In addition, in silico analysis performed in our study predicted not only both $\mathrm{R} 736 \mathrm{H}$ and $\mathrm{R} 882 \mathrm{H}$ variations in catalytic domain but also a novel E177V variation in regulatory domain to be deleterious to protein function, and previously known mutation hotspot R882 was not frequently detected in our present study. These results can be novel findings of our present study since 8822 residue of DNMT3A gene has been regarded as a frequently mutated hotspot in previous studies [20,21,24-27], which would have a gainof-function activity or dominant negative effects [20]. All these results may suggest the presence of ethnic variation in the characteristics of DNMT3A mutations and justify the application of detection method which covers all exons of DNMT3A gene rather than mutation hotspot R882 in Korean population.

Our structural analysis using 3D model showed that R736 residue is exposed to the DNMT3L molecules that are a stimulating factor of DNMT3A and a mediator of transcriptional repression through interaction with histone deacetylase 1 [30]. Therefore, it may be considered that the alteration of R736 residue may affect the efficiency and regulation of DNMT3A protein function, as similarly with previously demonstrated $\mathrm{R} 882$ residue [21]. In contrast, in the regulatory domain of DNMT3A protein, only E177V was identified as deleterious mutation and we could not provide the possible explanation for this result in our present study. More extensive functional analysis on the DNMT3A protein should be required for the clarification of this finding.

DNMT3A mutations have been reported to occur in patients with NPM1 and FLT3 mutations [24, 25, 27] but the clinical and prognostic impact of DNMT3A mutations has not been confirmed yet. Some studies revealed the poor prognostic effect of DNMT3A mutations on survival outcome, both in overall [25] and in high risk (FLT3 ITD mutated but NPM1 nonmutated) NK AML patients [24]. Two studies performed in Asian population also reported poor prognostic impact of DNMT3A mutations in NK AML $[28,29]$. In contrast, other studies also reported no significant prognostic effect of DNMT3A mutations in AML patients with intermediate risk group [27] and in low risk (NPM1 mutated but FLT3 ITD unmutated) NK AML patients [24]. A recent study reported that the poor prognostic relevance 
TABLE 4: Multivariate analysis of overall survival.

\begin{tabular}{|c|c|c|c|}
\hline \multirow{2}{*}{ Variables } & \multicolumn{3}{|c|}{ Overall survival } \\
\hline & HR $(95 \% \mathrm{CI})$ & $P$ value & Prognostic impact \\
\hline \multicolumn{4}{|l|}{ All 67 patients } \\
\hline FLT3 ITD mutation (compared with wild type) & $2.798(1.041-7.517)$ & 0.041 & Poor \\
\hline \multicolumn{4}{|l|}{ DNMT3A mutation (compared with wild type) } \\
\hline Nonsynonymous variations & $1.229(0.391-3.864)$ & 0.724 & $\mathrm{NS}^{*}$ \\
\hline Deleterious mutations & $2.285(0.459-11.374)$ & 0.313 & $\mathrm{NS}^{*}$ \\
\hline R882 mutation & $2.285(0.459-11.374)$ & 0.313 & $\mathrm{NS}^{*}$ \\
\hline Increasing age & $1.023(0.991-1.055)$ & 0.157 & NS \\
\hline Increrasing BM blasts & $1.018(0.986-1.051)$ & 0.285 & NS \\
\hline SCT performed (compared to not performed) & $0.118(0.035-0.399)$ & 0.001 & Good \\
\hline
\end{tabular}

Note: $P$ values were adjusted with FLT3 ITD mutation status, age, BM blast percentage, and stem cell transplantation performance status (in all 67 patients ${ }^{*}$ ). FLT3: fms-related tyrosine kinase 3; ITD: internal tandem duplications; DNMT3A: DNA methyltransferase 3A; R: arginine; BM: bone marrow; SCT: stem cell transplantation; HR: hazard ratio; CI: confidence interval; NS: not significant.

of DNMT3A mutation is dependent on age and its mutation type (R882 versus non-R882) [26]. Our present study could also demonstrate a trend of association between FLT3 ITD mutation and DNMT3A mutations in terms of mutation frequency, as reported in the previous literature $[24,25,27]$. However, our present study could not demonstrate a significant effect of DNMT3A mutations on both clinical features and prognosis, regardless of mutation type (nonsynonymous variations, deleterious mutations, or R882 mutation hotspot) in Korean NK AML patients although the trend was toward adverse prognosis. This result may support previously given hypothesis in our present study referring the presence of ethnic variation in the characteristics of DNMT3A mutations. In addition, from our present study result it can be speculated that the adverse prognostic effect of DNMT3A mutations may not exist in overall Korean NK AML patients and although some variations are predicted to be deleterious to protein function in our study, the adverse prognostic impact may reside in all non-synonymous variations of DNMT3A gene and, therefore, all 23 exons of DNMT3A gene should be screened for the detection of clinically important DNMT3A mutations.

Our study has some important limitations in terms of small patient numbers in each subgroup and mutation analysis of other genes with clinical significance. First, our study cohort included total 67 patients (39 in first and 28 in second study cohort) who were diagnosed and treated in single tertiary hospital, and statistical power in the comparison of clinical features and prognosis with respect to DNMT3A mutation status, especially in the analysis focused on FLT3 ITD mutated patients, is estimated to be significantly low. Therefore, our present results should be interpreted with caution and regarded to be preliminary data providing future issues to be addressed. Second, although both NPM1 and CEBPA mutations have prognostic value in NK AML and some previous study also suggested the association of NPM1 and DNMT3A mutations [24, 25, 27], our present study did not include the data of NPM1 and CEBPA mutation status and, therefore, comprehensive analysis in terms of mutational relations among three genes (NPM1, CEBPA, and $D N M T 3 A$ ) could not be performed. More large scaled and comprehensive study with these data should be required in the future to address these points.

\section{Conclusions}

Our present study identified three novel (I158M, E177V, and $\mathrm{K} 219 \mathrm{~V}$ in the regulatory domain) and two known (R736H and $\mathrm{R} 882 \mathrm{H}$ in the methyltransferase domain) DNMT3A nonsynonymous variations, and three (E177V, R736H and R882H) of them were predicted to be deleterious to protein function and structure. The incidences of DNMT3A nonsynonymous variations, deleterious mutations, and R882 mutation were $14.9 \%-17.9 \%, 10.3 \%-10.4 \%$, and $7.5 \%$, respectively, in Korean NK AML patients, without predominance of known mutation hotspot R882. DNMT3A mutations were frequently detected in FLT3 ITD mutated NK AML patients but did not significantly affect clinical features and prognosis in Korean NK AML patients, and this issue needs to be further evaluated in the future.

\section{Conflict of Interests}

The authors declare that there is no conflict of interests regarding the publication of this paper.

\section{Acknowledgments}

This study was supported by Biomedical Research Institute and Pusan Cancer Center Grant (2011-30), Pusan National University Hospital.

\section{References}

[1] J. C. Byrd, K. Mrózek, R. K. Dodge et al., "Pretreatment cytogenetic abnormalities are predictive of induction success, cumulative incidence of relapse, and overall survival in adult patients with de novo acute myeloid leukemia: results from cancer and leukemia group B (CALGB 8461)," Blood, vol. 100, no. 13, pp. 4325-4336, 2002.

[2] D. Grimwade, H. Walker, F. Oliver et al., "The importance of diagnostic cytogenetics on outcome in AML: analysis of 
1,612 patients entered into the MRC AML 10 trial. The Medical Research Council Adult and Children's Leukaemia Working Parties," Blood, vol. 92, pp. 2322-2333, 1998.

[3] M. R. O’Donnell, C. N. Abboud, J. Altman et al., "Acute myeloid leukemia," Journal of the National Comprehensive Cancer Network, vol. 10, no. 8, pp. 984-1021, 2012.

[4] S. P. Whitman, K. J. Archer, L. Feng et al., "Absence of the wild-type allele predicts poor prognosis in adult de novo acute myeloid leukemia with normal cytogenetics and the internal tandem duplication of FLT3: a cancer and leukemia group B study," Cancer Research, vol. 61, no. 19, pp. 7233-7239, 2001.

[5] Y. Yamamoto, H. Kiyoi, Y. Nakano et al., "Activating mutation of D835 within the activation loop of FLT3 in human hematologic malignancies," Blood, vol. 97, no. 8, pp. 2434-2439, 2001.

[6] B. Kainz, D. Heintel, R. Marculescu et al., "Variable prognostic value of FLT3 internal tandem duplications in patients with de novo AML and a normal karyotype, $\mathrm{t}(15 ; 17), \mathrm{t}(8 ; 21)$ or inv(16)," The Hematology Journal, vol. 3, no. 6, pp. 283-289, 2002.

[7] S. P. Whitman, K. Maharry, M. D. Radmacher et al., "FLT3 internal tandem duplication associates with adverse outcome and gene- and microRNA-expression signatures in patients 60 years of age or older with primary cytogenetically normal acute myeloid leukemia: a cancer and leukemia group B study," Blood, vol. 116, no. 18, pp. 3622-3626, 2010.

[8] S. P. Whitman, A. S. Ruppert, M. D. Radmacher et al., "FLT3 D835/I836 mutations are associated with poor disease-free survival and a distinct gene-expression signature among younger adults with de novo cytogenetically normal acute myeloid leukemia lacking FLT3 internal tandem duplications," Blood, vol. 111, no. 3, pp. 1552-1559, 2008.

[9] S. Schnittger, C. Schoch, W. Kern et al., "Nucleophosmin gene mutations are predictors of favorable prognosis in acute myelogenous leukemia with a normal karyotype," Blood, vol. 106, no. 12, pp. 3733-3739, 2005.

[10] K. Döhner, R. F. Schlenk, M. Habdank et al., "Mutant nucleophosmin (NPM1) predicts favorable prognosis in younger adults with acute myeloid leukemia and normal cytogenetics: interaction with other gene mutations," Blood, vol. 106, no. 12, pp. 3740-3746, 2005.

[11] R. G. W. Verhaak, C. S. Goudswaard, W. van Putten et al., "Mutations in nucleophosmin (NPM1) in acute myeloid leukemia (AML): association with other gene abnormalities and previously established gene expression signatures and their favorable prognostic significance," Blood, vol. 106, no. 12, pp. 3747-3754, 2005.

[12] S. Fröhling, R. F. Schlenk, I. Stolze et al., "CEBPA mutations in younger adults with acute myeloid leukemia and normal cytogenetics: prognostic relevance and analysis of cooperating mutations," Journal of Clinical Oncology, vol. 22, no. 4, pp. 624633, 2004.

[13] C. L. Green, K. K. Koo, R. K. Hills, A. K. Burnett, D. C. Linch, and R. E. Gale, "Prognostic significance of CEBPA mutations in a large cohort of younger adult patients with acute myeloid leukemia: impact of double CEBPA mutations and the interaction with FLT3 and NPM1 mutations," Journal of Clinical Oncology, vol. 28, no. 16, pp. 2739-2747, 2010.

[14] C. Preudhomme, C. Sagot, N. Boissel et al., "Favorable prognostic significance of CEBPA mutations in patients with de novo acute myeloid leukemia: a study from the Acute Leukemia French Association (ALFA)," Blood, vol. 100, no. 8, pp. 27172723,2002 .
[15] S. H. Park, H. S. Chi, Y. U. Cho, S. Jang, and C. J. Park, “CEBPA single mutation can be a possible favorable prognostic indicator in NPM1 and FLT3-ITD wild-type acute myeloid leukemia patients with intermediate cytogenetic risk," Leukemia Research, vol. 37, no. 11, pp. 1488-1494, 2013.

[16] S. Abbas, S. Lugthart, F. G. Kavelaars et al., "Acquired mutations in the genes encoding IDH1 and IDH2 both are recurrent aberrations in acute myeloid leukemia: prevalence and prognostic value," Blood, vol. 116, no. 12, pp. 2122-2126, 2010.

[17] P. Paschka, R. F. Schlenk, V. I. Gaidzik et al., "IDH1 and IDH2 mutations are frequent genetic alterations in acute myeloid leukemia and confer adverse prognosis in cytogenetically normal acute myeloid leukemia with NPM1 mutation without FLT3 internal tandem duplication," Journal of Clinical Oncology, vol. 28, no. 22, pp. 3636-3643, 2010.

[18] H.-Y. Chao, Z.-X. Jia, T. Chen et al., "IDH2 mutations are frequent in Chinese patients with acute myeloid leukemia and associated with NPM1 mutations and FAB-M2 subtype," International Journal of Laboratory Hematology, vol. 34, no. 5, pp. 502-509, 2012.

[19] F. Ravandi, K. Patel, R. Luthra et al., "Prognostic significance of alterations in IDH enzyme isoforms in patients with AML treated with high-dose cytarabine and idarubicin," Cancer, vol. 118, no. 10, pp. 2665-2673, 2012.

[20] T. J. Ley, L. Ding, M. J. Walter et al., "DNMT3A mutations in acute myeloid leukemia," The New England Journal of Medicine, vol. 363, no. 25, pp. 2424-2433, 2010.

[21] X.-J. Yan, J. Xu, Z.-H. Gu et al., "Exome sequencing identifies somatic mutations of DNA methyltransferase gene DNMT3A in acute monocytic leukemia," Nature Genetics, vol. 43, no. 4, pp. 309-315, 2011.

[22] M. A. McDevitt, "Clinical applications of epigenetic markers and epigenetic profiling in myeloid malignancies," Seminars in Oncology, vol. 39, no. 1, pp. 109-122, 2012.

[23] J. J. Trowbridge, J. W. Snow, J. Kim, and S. H. Orkin, "DNA methyltransferase 1 is essential for and uniquely regulates hematopoietic stem and progenitor cells," Cell Stem Cell, vol. 5, no. 4, pp. 442-449, 2009.

[24] F. Thol, F. Damm, A. Lüdeking et al., "Incidence and prognostic influence of DNMT3A mutations in acute myeloid leukemia," Journal of Clinical Oncology, vol. 29, no. 21, pp. 2889-2896, 2011.

[25] A. Renneville, N. Boissel, O. Nibourel et al., "Prognostic significance of DNA methyltransferase $3 \mathrm{~A}$ mutations in cytogenetically normal acute myeloid leukemia: a study by the Acute Leukemia French Association," Leukemia, vol. 26, no. 6, pp. 1247-1254, 2012.

[26] G. Marcucci, K. H. Metzeler, S. Schwind et al., "Age-related prognostic impact of different types of DNMT3A mutations in adults with primary cytogenetically normal acute myeloid leukemia," Journal of Clinical Oncology, vol. 30, no. 7, pp. 742750, 2012.

[27] J. Markova, P. Michkova, K. Burckova et al., "Prognostic impact of DNMT3A mutations in patients with intermediate cytogenetic risk profile acute myeloid leukemia," European Journal of Haematology, vol. 88, no. 2, pp. 128-135, 2012.

[28] Y. Shen, Y. M. Zhu, X. Fan et al., "Gene mutation patterns and their prognostic impact in a cohort of 1185 patients with acute myeloid leukemia," Blood, vol. 118, no. 20, pp. 5593-5603, 2011.

[29] H.-A. Hou, Y.-Y. Kuo, C.-Y. Liu et al., "DNMT3A mutations in acute myeloid leukemia: stability during disease evolution and clinical implications," Blood, vol. 119, no. 2, pp. 559-568, 2012. 
[30] Y. Wang, L. Y. Geer, C. Chappey, J. A. Kans, and S. H. Bryant, "Cn3D: sequence and structure views for Entrez," Trends in Biochemical Sciences, vol. 25, no. 6, pp. 300-302, 2000.

[31] M. S. Kim, Y. R. Kim, N. J. Yoo, and S. H. Lee, "Mutational analysis of DNMT3A gene in acute leukemias and common solid cancers," APMIS, vol. 121, no. 2, pp. 85-94, 2013. 


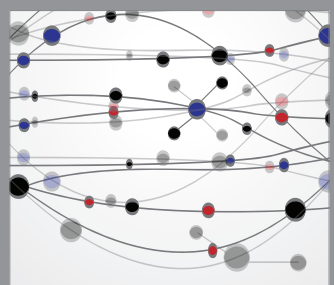

The Scientific World Journal
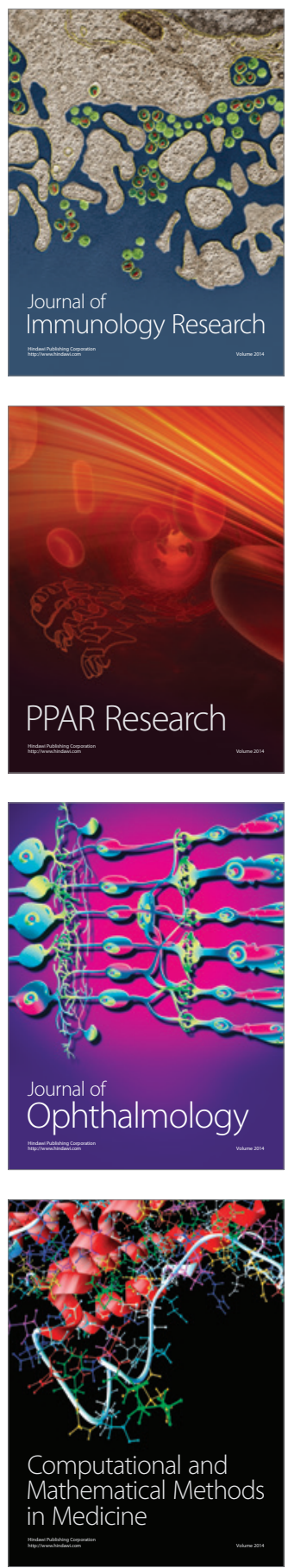

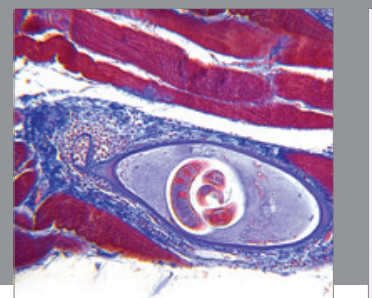

Gastroenterology

Research and Practice
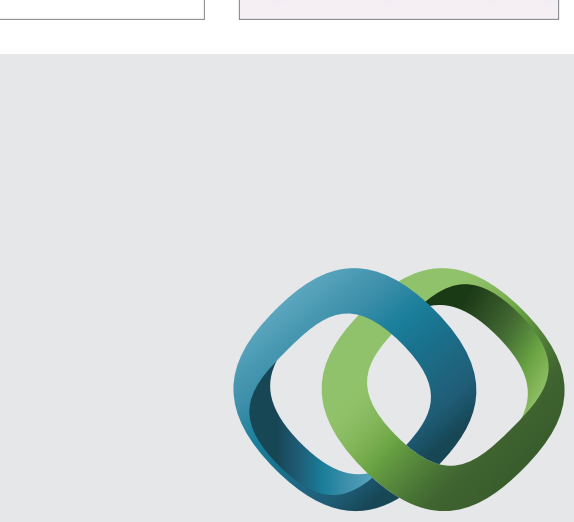

\section{Hindawi}

Submit your manuscripts at

http://www.hindawi.com
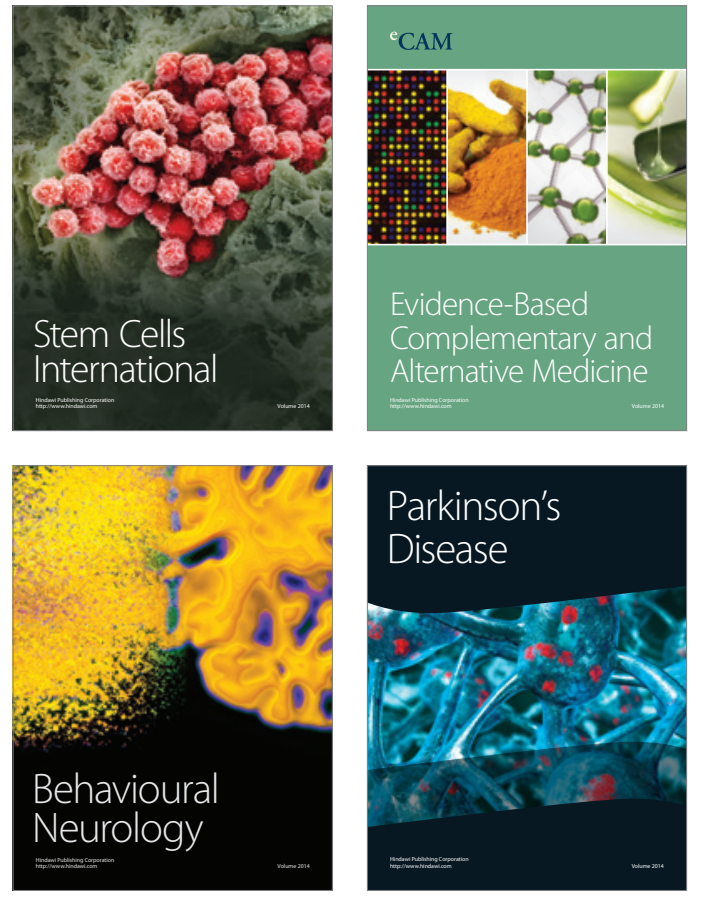
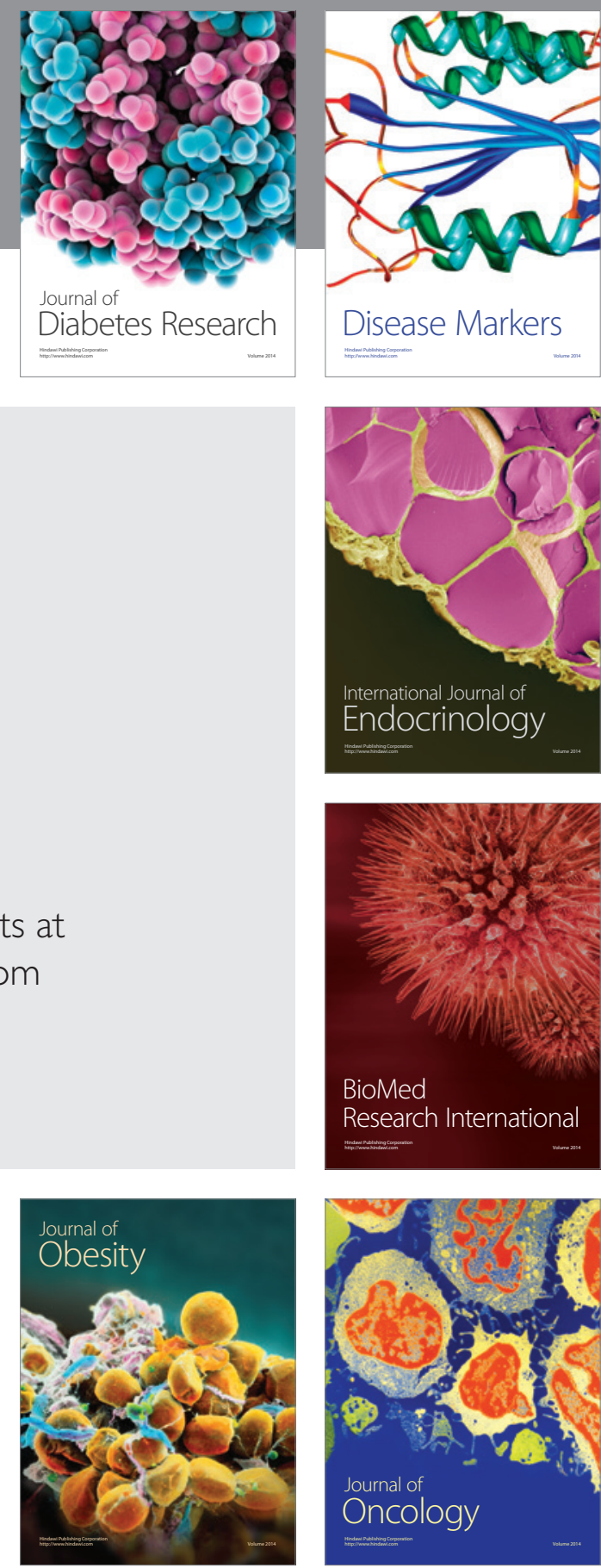

Disease Markers
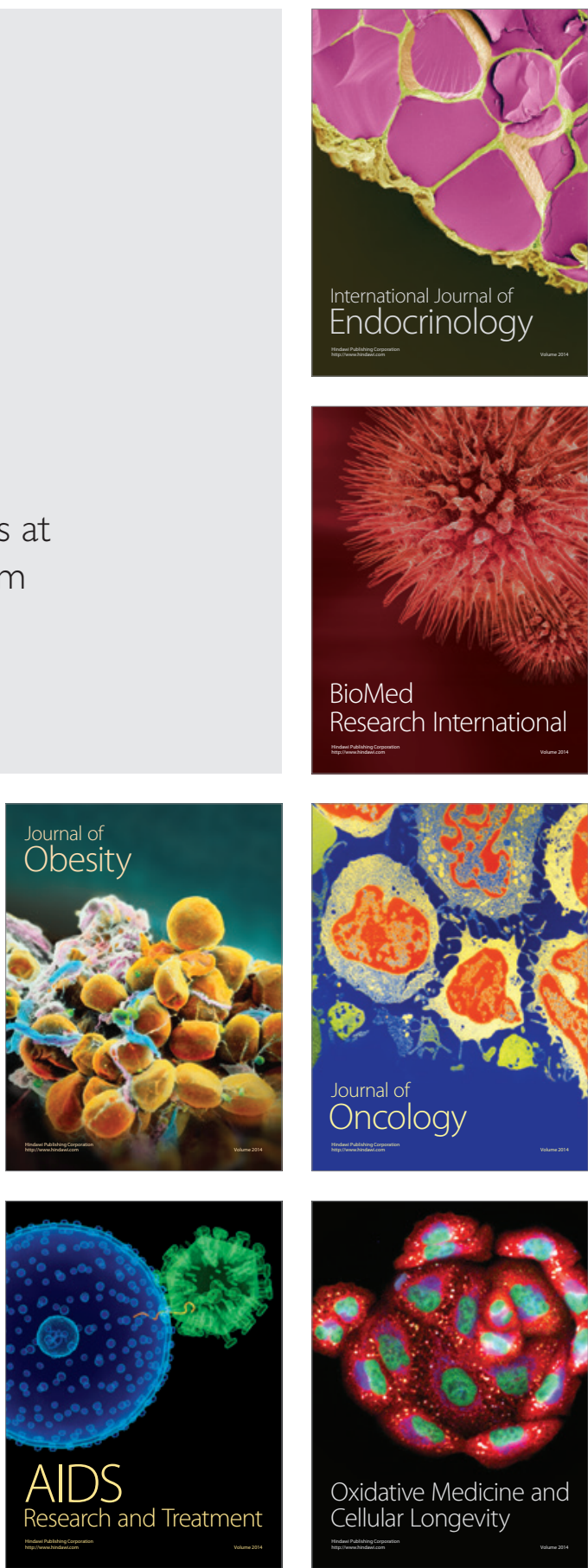